\title{
Water Dimers in the Atmosphere II: Results from the VRT(ASP-W)III Potential Surface
}

\author{
Nir Goldman, ${ }^{\dagger, \pi}$ Claude Leforestier, ${ }^{\S}$ and R. J. Saykally*, ${ }^{*}$ \\ Department of Chemistry, University of California, Berkeley, California 94720-1416, and Laboratoire Structure \\ et Dynamique des Systèmes Moléculaire et Solides (UMR 5363), CC 014, Université des Sciences et \\ Techniques du Langue-doc, 34095 Montpellier Cédex, France
}

Received: May 16, 2003; In Final Form: September 30, 2003

\begin{abstract}
We report refined results for the equilibrium constant for water dimerization $\left(K_{\mathrm{P}}\right)$, computed as a function of temperature via fully coupled 6D calculation of the canonical $\left(\mathrm{H}_{2} \mathrm{O}\right)_{2}$ partition function on VRT(ASP-W)III, the most accurate water dimer potential energy surface currently available. Partial pressure isotherms calculated for a range of temperatures and relative humidities indicate that water dimers can exist in sufficient concentrations (e.g., $10^{18} \mathrm{~m}^{-3}$ at $30^{\circ} \mathrm{C}$ and $100 \%$ relative humidity) to affect physical and chemical processes in the atmosphere. The determinations of additional thermodynamic properties $\left(\Delta G, \Delta H, \Delta S, C_{P}\right.$, and $\left.C_{V}\right)$ for $\left(\mathrm{H}_{2} \mathrm{O}\right)_{2}$ are presented, and the role of quasi-bound states in the calculation of $K_{\mathrm{P}}$ is discussed at length.
\end{abstract}

\section{Introduction}

The role of water dimers in the atmosphere is an important and controversial issue that has not been resolved. Proposed contexts include the excess atmospheric absorption of solar radiation, ${ }^{1-3}$ which has been ascribed to the water dimer, "the water continuum" absorption in the far-infrared, ${ }^{4}$ and catalysis of the formation of atmospheric $\mathrm{H}_{2} \mathrm{SO}_{4},{ }^{5,6}$ which has has been confirmed by experimental results in the laboratory. ${ }^{7,8}$ In addition, the abundance of water dimers in the atmosphere may be important for modeling water condensation ${ }^{9}$ and for the formation of radical complexes such as $\mathrm{HO}_{2} \cdot \mathrm{H}_{2} \mathrm{O}$, which have been predicted to exist in relatively high concentrations in the atmosphere. ${ }^{10,11}$ As shown in the seminal work by Vaida et al., ${ }^{12}$ the search for such a water dimer spectroscopic signature in the atmosphere requires a highly accurate determination of its concentrations. Nevertheless and despite various experimental and theoretical attempts, there remains considerable uncertainty in atmospheric water dimer concentrations, ${ }^{2,9,13-16}$ primarily because generally only relatively crude harmonic dynamics models have been used to describe the highly anharmonic dimer and experimental results depend heavily on these theoretical predictions of the absorption wavelengths. One exception to the above is the work of Evans and Vaida, wherein they model the anharmonicity of the water dimer and larger clusters via Wertheim's analytically simple theory of association. ${ }^{17}$ While their results compare well with earlier work using a harmonic dimer approximation ${ }^{16}$ and the simplicity of the model is compelling, their approach contains several significant limitations, including the fact that it is very orientationally restrictive and too simple to provide quantitatively accurate results. Only recently have rigorous models for the dimer potential and rigorous methods for computing concentrations from them been developed. ${ }^{18-20}$ Recent measurements ${ }^{2,21}$ have shown that the absorption of solar flux at dimer vibrational overtone wavelengths predicted in ref 2 is negligibly small. ${ }^{1,21}$ However, the

\footnotetext{
* Corresponding author. E-mail: saykally@uclink4.berkeley.edu.

$\dagger$ University of California.

II Present address: Lawrence Livermore National Laboratory, Chemistry and Materials Science Directorate, L-268, Livermore, CA 94551.

$\S$ Université Sciences et Techniques du Langue-doc.
}

very recent article by Pfeilsticker et al. ${ }^{22}$ reports measured dimer atmospheric absorption the intensities of which are in agreement with values determined via a combination of calculated water dimer line strengths by Low and Kjaergaard ${ }^{23}$ and the classic laboratory experiments of Curtiss et al. ${ }^{24}$ Their results are lower than absorption intensities determined using our results for $K_{\mathrm{P}}$ calculated on the original VRT(ASP-W) potential. ${ }^{25}$ In addition, in a recent publication in this journal, Schenter et al. ${ }^{26}$ argue that our original calculation of water dimer atmospheric concentrations were probably too low because of the exclusion of long-lived quasi-bound states.

Assuming rigid monomers, the water dimer potential is a six dimensional surface with a very complex topology, namely, eight identical global minima connected by three different low barrier tunneling paths. ${ }^{27}$ Thus, while many ab initio and empirical pair potentials have been published, none were able to accurately describe the observed dimer properties. ${ }^{28} \mathrm{Ab}$ initio potentials generally suffer from basis set superposition error or poor convergence of the interaction energy or both, and empirical potentials are usually designed to mimic the bulk phase, which leads to poor estimations of the dipole moment and binding energy of the dimer.

In this paper, we determine $K_{\mathrm{P}}(T)$ via the canonical partition function and subsequently calculate the dimer partial pressure versus relative humidity using the recently determined VRT(ASP-W)III water dimer intermolecular potential energy surface (IPS). ${ }^{20}$ This is the third fitting of Millot and Stone's ASP-W ab initio potential ${ }^{29}$ to $\left(\mathrm{D}_{2} \mathrm{O}\right)_{2}$ microwave and far-infrared (FIR) vibration-rotation-tunneling (VRT) transitions, which constitute a far more extensive data set than those available for $\left(\mathrm{H}_{2} \mathrm{O}\right)_{2}$. The dimer tunneling splittings from hydrogen bond rearrangements and the intermolecular vibrational frequencies provide a sensitive probe of the complex water IPS, ${ }^{30,31}$ and such measurements have been made extensively by our laboratory. ${ }^{32-34}$ The VRT(ASP-W)III potential was generated by fitting six of the exchange-repulsion parameters in ASP-W ${ }^{29}$ to $\left(\mathrm{D}_{2} \mathrm{O}\right)_{2}$ microwave and far-IR transitions using a nonlinear least-squares regression procedure. ${ }^{20}$ The ASP-W potential has 72 parameters, but it was found previously that accurate fits could be produced by varying only a few of the 
TABLE 1: Spin Statistical Weights for $\left(\mathrm{H}_{2} \mathrm{O}\right)_{2}$ and for $\left(\mathrm{D}_{2} \mathrm{O}\right)_{2}$

\begin{tabular}{|c|c|c|c|c|c|}
\hline level symmetry & $A_{1}^{ \pm}$ & $A_{2}{ }^{ \pm}$ & $B_{1}^{ \pm}$ & $B_{2}{ }^{ \pm}$ & $E^{ \pm}$ \\
\hline$\left(\mathrm{H}_{2} \mathrm{O}\right)_{2}$ weight & 1 & 3 & 0 & 6 & 3 \\
\hline$\left(\mathrm{D}_{2} \mathrm{O}\right)_{2}$ weight $^{a}$ & 6 & 15 & 3 & 21 & 18 \\
\hline
\end{tabular}

exchange-repulsion parameters. ${ }^{18}$ VRT(ASP-W)III is a substantial improvement over the original VRT(ASP-W) potential and is the most accurate water pair potential determined to date, although van der Avoird and co-workers have obtained one of comparable quality for the $\left(\mathrm{H}_{2} \mathrm{O}\right)_{2}$ isotopomer by "tuning" an $\mathrm{ab}$ initio potential derived from symmetry-adapted perturbation theory (SAPT). ${ }^{35}$ The $K_{\mathrm{P}}(T)$ calculations presented below represent a refinement to those previously published by us, ${ }^{25}$ which utilized the original VRT(ASP-W) surface, ${ }^{18}$ in that the VRT transitions produced by VRT(ASP-W)III compare much more closely with experimental values. ${ }^{32,33}$ In addition, we present the results of a number of other $\left(\mathrm{H}_{2} \mathrm{O}\right)_{2}$ thermodynamic properties calculated from VRT(ASP-W)III, including $\Delta G, \Delta H$, $\Delta S, C_{P}$, and $C_{V}$. Finally, the role of quasi-bound states in our calculations will be quantified and discussed.

\section{Methods}

Calculation of $\boldsymbol{K}_{\mathrm{P}}(\boldsymbol{T})$. As described previously, ${ }^{25}$ the computationally demanding calculations of VRT states on the VRT(ASP-W)III potential necessary to determine $K_{\mathrm{P}}(T)$ were performed using the pseudospectral split hamiltonian (PSSH) approach. ${ }^{19,36}$ In PSSH, the radial (R) dependence of the potential is represented by a basis of sine functions, optimized via the HEG contraction, ${ }^{37}$ and the angular part by a set of Wigner functions. Improvements were made on the original PSSH code by including the ARPACK Lanczos matrix package, which keeps the Lanczos vectors orthogonal to avoid possible spurious eigenvalues. The computational difficulties associated with such a $6 \mathrm{D}$ calculation are formidable. It was found that ca. 10 basis functions were needed per degree of freedom (six) to achieve acceptable accuracy. As a result, converging a partition function can take ca. 1 week of CPU time on a Dell Precision 420 workstation with a $733 \mathrm{MHz}$ Pentium III processor. In addition, storage of the Lanczos basis vectors, required to keep orthogonality, generally requires close to 512 $\mathrm{MB}$ of memory. For our purposes, the primitive R-basis for the calculation was set to 16 sine functions and 19 grid points (with 4 points kept after HEG optimization) over a range of 4-7 bohr units, and $j_{\max }$ for the monomer was set to seven. This grid size and radial range, smaller than what was previously used, ${ }^{25}$ was ultimately chosen because it adequately samples the VRT(ASP-W)III potential without making the calculation of eigenstates too expensive. In addition, the radial grid limits define an $\mathrm{O}-\mathrm{O}$ length for the dimer that is physically intuitive. Convergence was again estimated by increasing the radial grid to as large as 20 radial functions and 23 points (keeping $4-6$ HEG points) over a range of 4-25 bohr and separately by extending the angular grid to as high as $j_{\max }=9$, wherein the equilibrium constant was found to not vary by more than $5 \%$. This indicates that the chosen grid size and radial range is sufficient to accurately sample the potential surface.

The method used for calculation of $K_{\mathrm{P}}(T)$ is summarized in detail below. Eigenvalues for $\left(\mathrm{H}_{2} \mathrm{O}\right)_{2}$ with $J=0$ were calculated to energies near the dissociation limit, which was determined to be the lowest eigenvalue computed for the $A_{1}{ }^{+}$symmetry (approximately $-1071 \mathrm{~cm}^{-1}$ for VRT(ASP-W)III for the grid size and radial range used for our calculations). The water dimer was then assumed to be a prolate symmetric top, and the computed VRT eigenvalues were used to calculate a partition function in the canonical ensemble according to

$$
Q_{\text {dimer }}=g(\operatorname{sym}, J, K) \sum_{n} \mathrm{e}^{-\beta E_{n}}
$$

Here $g(\operatorname{sym}, J, K)$ is the degeneracy due to the spin statistical weight of the symmetry for which the eigenstates have been calculated and that due to the $K$ and $M$ levels for a prolate top, $\beta=k_{\mathrm{B}} T$, and $E_{n}$ are the computed eigenvalues. The spin statistical weights are well-known ${ }^{38}$ (see Table 1), and the degeneracy for a prolate top is

$$
g(J, K)= \begin{cases}(2 J+1) & \text { for } K=0 \\ 2(2 J+1) & \text { for } K>0\end{cases}
$$

To efficiently compute the $J>0$ energy levels for $\left(\mathrm{H}_{2} \mathrm{O}\right)_{2}$, we employed a technique similar to $J$-shifting ${ }^{39-41}$ to approximate the rotational energy of the dimer. The prolate top rotational energy level expression

$$
F(J, K)=h c\left[B J(J+1)+(A-B) K^{2}\right]
$$

was used, where $F(J, K)$ is the rotational energy level correction to the computed eigenvalues, $h$ is Plank's constant, and $c$ is the speed of light. The same rotational constants used previously, ${ }^{25}$ $A=7.5919 \mathrm{~cm}^{-1}, B=0.2047 \mathrm{~cm}^{-1}, C=0.2039 \mathrm{~cm}^{-1}$, were used again in this calculation. Once again, these rotational constants show that the prolate top is indeed a good vibrational ground-state approximation for the water dimer.

To summarize, our canonical partition function calculation goes as follows: (1) Calculation of a vibrational line list for $J$ $=0$ using the PSSH code with ARPACK Lanczos is performed. Eigenvalues were calculated up to ca. $0 \mathrm{~cm}^{-1}$, which necessitated the calculation of ca. 400 eigenvalues per symmetry. (2) Using the above line list, we calculate the partition function with the equilibrium rotational constants by applying eq 3 to determine the $J>0$ states. To make the problem more tractable, rotational constants are assumed to be constant throughout the calculation. In addition, any calculated rovibrational state above dissociation is discarded. Each calculation is performed at a predefined temperature. (3) The partition function is converged for each symmetry separately to within a tolerance of $10^{-3}$. In other words, we calculate the contribution from $\mathrm{A}_{1}{ }^{+}$, followed by that from $A_{1}{ }^{-}$, etc. $B_{1}^{ \pm}$states are neglected due to the 0 spin statistical weight.

Once the dimer partition partition function has been determined, calculation of the dimerization equilibrium constant $\left(K_{2}\right)$ follows from the canonical expression

$$
K_{2}=\frac{\left(Q_{2} / \lambda_{2}{ }^{3}\right)}{\left(Q_{1} / \lambda_{1}{ }^{3}\right)^{2}}
$$

where $Q_{1}$ and $Q_{2}$ are the monomer and dimer partition functions, respectively, and

$$
\lambda_{i}=h /\left(2 \pi m_{i} k_{\mathrm{B}} T\right)^{1 / 2}
$$

where $h$ is Planck's constant, $k_{\mathrm{B}}$ is the Boltzman constant, and $T$ is the absolute temperature. $K_{2}$ can be converted to $K_{\mathrm{P}}(T)$ [atm ${ }^{-1}$ ] by dividing by the appropriate value of $R T$, where $R$ is the universal gas constant.

The monomer partition function $\left(Q_{1}\right)$ used is identical to that used previously. ${ }^{25}$ The monomer partition function developed 
by Harris et al. ${ }^{42}$ uses the VT2 line list calculated by Viti and Tennyson $^{43}$ by performing explicit dynamics on the experimentally determined water monomer PJT2 potential energy surface of Polansky, Jensen, and Tennyson. ${ }^{44}$ PJT2 was used to generate energy levels for all rotational states with up to $J=35$, which resulted in a list of about 200000 rotational-vibrational energy levels. The PJT2 potential is quite accurate in determining highenergy rovibrational states of the monomer because it reproduced all of the experimentally determined energy levels with up to $J=14$ known at the time of its construction to within a standard deviation of only $0.6 \mathrm{~cm}^{-1}$. The values of $Q_{1}$ published in ref 42 were plotted over a temperature range of 100-1500 $\mathrm{K}$, and the resulting curve was fit via a least-squares routine to a fifth-order polynomial to obtain an analytical expression.

\section{Symmetry Considerations}

At this point, it is worthwhile to make a brief comment on the symmetry of the rovibrational states of the dimer. In starting from a vibrational state with $(J=0, K=0)$, in generating the corresponding $\left(J^{\prime}, K=0\right)$ state, one must make the following symmetry change: ${ }^{45}$

$$
\begin{array}{ll}
\left.\begin{array}{ll}
E^{ \pm} \rightarrow E^{\mp} \\
\rightarrow B,+\rightarrow-
\end{array}\right\} & \text { if } \Delta J \text { is odd } \\
\text { no change } & \text { if } \Delta J \text { is even }
\end{array}
$$

Note that this is also true if one starts from a state $(J, K)$ and generates a state $\left(J^{\prime}, K\right)$ with $K>0$ due to the double degeneracy of the $K>0$ states. $^{46}$

Less clear is what change occurs in going from $(J, K=0)$ to $(J, K>0)$. However, once again, because of the double degeneracy of the $K>0$ energy levels, one could use the following:

$$
\begin{aligned}
& A_{1}^{+} \rightarrow A_{2}^{-} \text {and } B_{2}^{-} \\
& A_{1}^{-} \rightarrow A_{2}^{+} \text {and } B_{2}^{+} \\
& B_{1}^{+} \rightarrow A_{2}^{+} \text {and } B_{2}^{+} \\
& B_{1}^{-} \rightarrow A_{2}^{-} \text {and } B_{2}^{-} \\
& A_{2}^{+} \rightarrow A_{1}^{-} \text {and } B_{1}^{+} \\
& A_{2}^{-} \rightarrow A_{1}^{+} \text {and } B_{1}^{-} \\
& B_{2}^{+} \rightarrow A_{1}^{+} \text {and } B_{1}^{-} \\
& B_{2}^{-} \rightarrow A_{1}^{-} \text {and } B_{1}^{+} \\
& E^{ \pm} \rightarrow E^{\mp}
\end{aligned}
$$

It should also be noted that the above relation could only be verified by inspection of energy level diagrams in ref 46 for ( $J=0, K=0)$ up to $(J=0, K=2)$, making it difficult to assess its reliability.

Hence, partially because of the above ambiguity, it is difficult to define a strict $J$-shifting scheme for our IPS, and thus symmetry changes were not included in our calculation of rovibrational states, that is, if a vibrational state exists with $\mathrm{A}_{1}{ }^{+}$ symmetry at $J=0$, its spin statistical weight is unchanged through the course of evaluating its $J>0$ rotational energy contributions. It is unclear how much the changes in nuclear spin statistical weights would affect our results, but it is reasonable to expect that, considering the high value of $J$ needed to converge our calculations and the corresponding quasi-

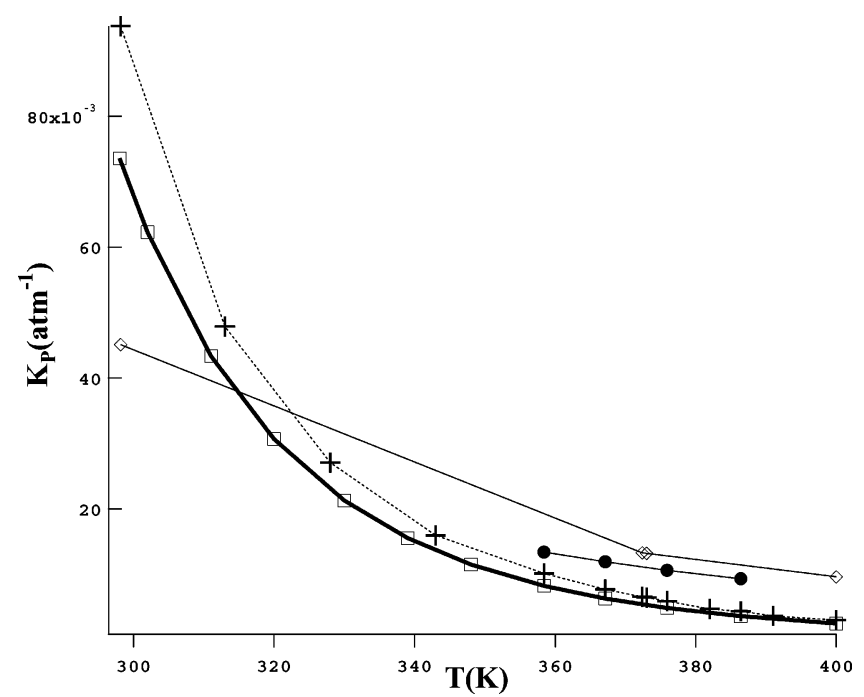

Figure 1. The temperature dependence of $K_{\mathrm{P}}$ using the monomer partition function developed by Harris et al. using the PJT2 monomer potential. ${ }^{42}$ The solid line with unshaded squares corresponds to calculations performed with the VRT(ASP-W)III potential and using the PJT2 potential monomer partition function, the dashed line with pluses $(+)$ corresponds to our original calculations,${ }^{25}$ the unshaded diamonds correspond to results from Slanina et al. using the MCY-B pair potential, ${ }^{47}$ and the solid circles corresponds to the values measured by Curtiss et al. ${ }^{24}$

degeneracy between these high-level rovibrational states of different symmetry (due to the flatness of the potential), that such symmetry effects will be minimal.

\section{Determination of Thermodynamic Properties}

$K_{\mathrm{P}}(\mathrm{T})$ and $\left(\mathrm{H}_{2} \mathrm{O}\right)_{2}$ Partial Pressure. After the monomer and dimer partition functions had been determined sufficiently well $\left(10^{-3}\right)$, calculations of $K_{\mathrm{P}}$ were performed over a large range of temperatures and compared with previously published theoretical and experimental results.

In Figure 1, the small number of points from Curtiss et al. ${ }^{24}$ is due to the limited temperature range over which their experiments were performed. As in our previous publication, ${ }^{25}$ extra points have been added in the plots of our calculations of the equilibrium constant to better display the exponential dependence of the curves. Although several harmonic calculations of $K_{\mathrm{P}}(T)$ exist (e.g., refs 2, 13, 14, and 16), we chose to compare to the results of Slanina et al. ${ }^{47}$ since theirs are determined over the largest temperature range. All of the calculations performed by Slanina et al. ${ }^{47}$ were done by finding the minimum of the potential via the analytic energy derivatives and then replacing this with a harmonic potential. The $K_{\mathrm{P}}$ measurements by Curtiss et al. were performed by using a modified thick hot wire cell and creating a voltage drop in the presence of $\mathrm{H}_{2} \mathrm{O}$ vapor. The voltage drop varies according to $1 / \lambda$, where $\lambda$ is the thermal conductivity of the sample. Measurements were made by varying the temperature and pressure of the sample and recording the thermal conductivity. The dimerization equilibrium constants were then determined at various temperatures by fitting the thermal conductivity values to theoretical expressions, which contain the dimerization constant as a parameter.

As found previously, ${ }^{25}$ a large discrepancy between our results and those of Slanina et al.9,47 is evident. This can be attributed to the fact that Slanina et al. used a simplistic harmonic potential to describe the dimer vibrations and did not account for the anharmonicity of the potential. Comparison of our $K_{\mathrm{P}}$ values with those obtained by Curtiss et al. show reasonable agreement, 


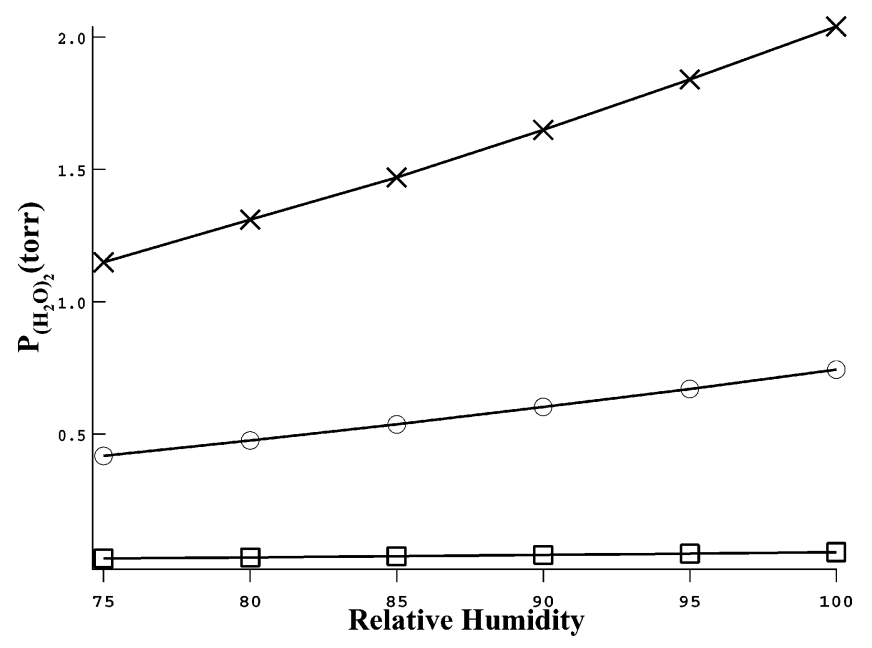

Figure 2. Comparison of $\left(\mathrm{H}_{2} \mathrm{O}\right)_{2}$ partial pressure vs relative humidity for temperatures from 298 to $358.4 \mathrm{~K}$. The squares correspond to a water dimer partial pressure profile at a temperature of $298 \mathrm{~K}$; the circles correspond to $T=339 \mathrm{~K}$; the crosses correspond to $T=358.4 \mathrm{~K}$.

although our results for the four temperatures at which Curtiss et al. made their measurements are considerably lower (ca. 20\%). Interestingly, our previous results for $K_{\mathrm{P}}(T),{ }^{25}$ performed with the first VRT(ASP-W) IPS, show much closer agreement to the results of Curtiss et al. This is likely an artifact of the original VRT(ASP-W) since VRT(ASP-W)III has proven to reproduce experimental water dimer data much more accurately. ${ }^{20}$ Also, it is important to note that by using their values for $\Delta H$ of dimerization, Curtiss et al. calculate a binding energy $\left(D_{\mathrm{e}}\right)$ of $-22.761 \pm 2.93 \mathrm{~kJ} / \mathrm{mol}$, which is considerably higher than the value found from either high-level ab initio calculations $(-20$ to $-21 \mathrm{~kJ} / \mathrm{mol})^{48-51}$ or from our VRT(ASP-W)III surface $(-20.078 \mathrm{~kJ} / \mathrm{mol})$, but again, a primitive harmonic approximation was used to estimate $D_{\mathrm{e}}$ from $\Delta H$. Hence, as we have stated, ${ }^{25}$ the $K_{\mathrm{P}}$ values measured by Curtiss et al. are an upper limit. Our results slightly underestimate the value of $K_{\mathrm{P}}$ because as the highly nonrigid $\mathrm{H}_{2} \mathrm{O}$ dimer accesses higher excited rotational states the average $\mathrm{O}-\mathrm{O}$ distance will increase, thus causing a decrease in the rotational constants, causing a corresponding increase in $K_{\mathrm{P}}$, and this effect is not included in our treatment. Again, experimenting with various grid sizes and ranges has shown that the equilibrium constant is relatively insensitive to the radial grid and range. For example, a calculation with a grid of seven radial functions over a range of 4-9 au yields an equilibrium constant that differs from that of a grid of 20 radial functions over a range of 4-15 au by only -1.6 at a temperature of $85.4{ }^{\circ} \mathrm{C}$. In any case, the agreement between our results and the experimental values determined by Curtiss et al. is reasonable over the very narrow temperature range of $30^{\circ} \mathrm{C}$ actually examined by them, but our calculations clearly span a much larger temperature range. We estimate the accuracy in our $K_{\mathrm{P}}$ values to be ca. $10 \%$ or better.

We also present a refined calculated dimer partial pressure vs relative humidity at several relevant temperatures in Figure 2. The results are quite close to what was found initially, ${ }^{25}$ and the analysis presented therein is summarized below. Slanina et al. greatly underestimate the value of the dimer partial pressure (Figure 3), while Curtiss et al. most probably overestimate this quantity (Figure 4). All data points were calculated by first finding the water monomer vapor pressure through calculating the saturated water vapor pressure using a variant of the Clausius-Clapeyron equation, $P=P_{0} \mathrm{e}^{-\Delta H /(R T)}$, and then by multiplying by the relative humidity. The values of the constants used in the equation were $P_{0}=7.51 \times 10^{8} \mathrm{mmHg}, \Delta H=42.8$

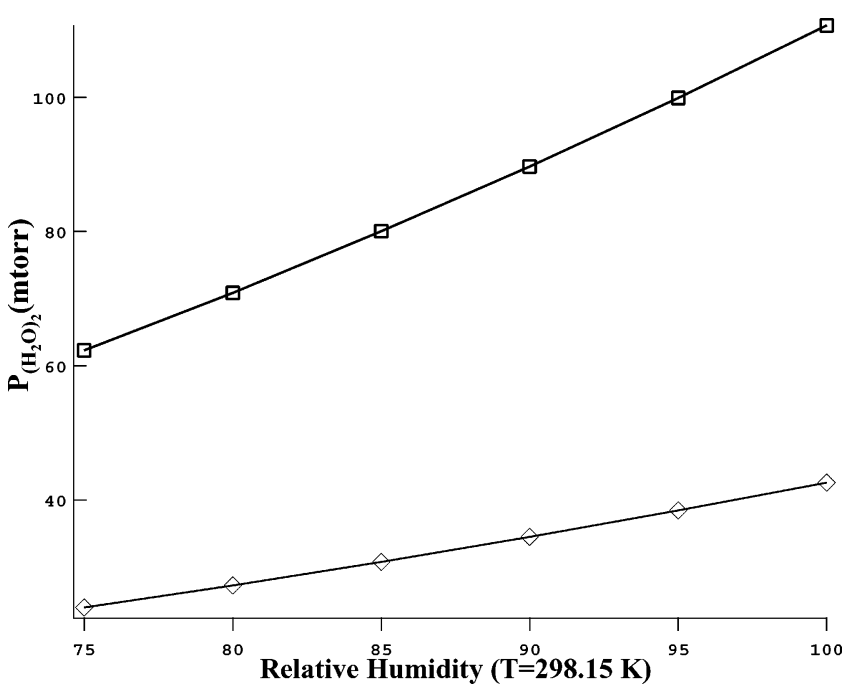

Figure 3. $\left(\mathrm{H}_{2} \mathrm{O}\right)_{2}$ partial pressures vs relative humidity at $T=298 \mathrm{~K}$, comparing calculations performed by Slanina et al. ${ }^{47}(\diamond)$ to our calculations performed with the VRT(ASP-W)III dimer potential ( $\square$ ).

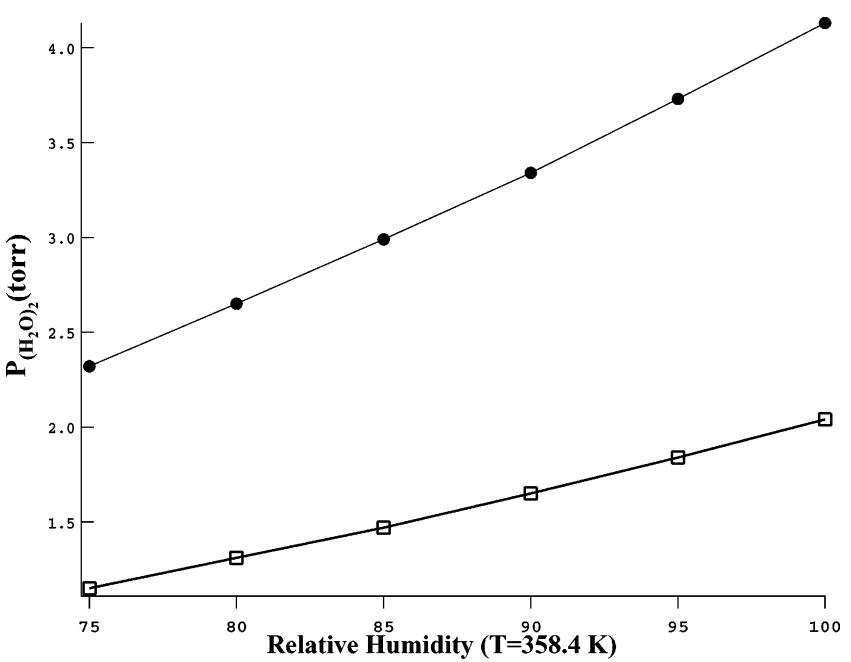

Figure 4. Dimer partial pressures vs relative humidity at $T=358.4$ $\mathrm{K}$, comparing the results of Curtiss et al. ( ) to our calculations with the VRT(ASP-W) dimer potential ( $\square$ ).

$\mathrm{kJ} / \mathrm{mol}$, and $R=8.314 \mathrm{~J} /(\mathrm{mol} \mathrm{K})$, the same as those used previously by us. ${ }^{25}$ The dimer partial pressure was then found by taking the dimerization equilibrium constant calculated at the temperature of interest and solving for the dimer partial pressure. Once again, it should be noted that the calculations of Slanina et al. were performed by finding the potential global minimum and making a harmonic approximation, which helps to explain the discrepancy between these results and ours. Also, it should be repeated that the dimer partial pressure derived from the results of Curtiss et al. should be considered an upper limit to the actual answer at the above temperature. From the results shown in Figure 2, wherein the water dimer partial pressure is found to be as large as 0.5 Torr at tropical conditions, it is probable that the water dimer can exist in the atmosphere in sufficient quantities to have significant effects on solar absorption, far-IR light propagation, the catalysis of $\mathrm{H}_{2} \mathrm{SO}_{4}$ formation, and other atmospheric processes.

To explain the absence of the water dimer $\mathrm{O}-\mathrm{H}$ vibrational overtone transitions in the atmospheric measurements of Daniel et al. ${ }^{1}$ over Boulder, CO, and Hill and Jones ${ }^{21}$ over Cambridge, England, we have once again recalculated the water dimer optical depth from our results. Using the atmospheric conditions of that day (partly cloudy with a surface dew point between 13 
and $16{ }^{\circ} \mathrm{C}$ ), their absorption cross section (taken from Tso et al. ${ }^{51}$ ), and our estimate of the $\left(\mathrm{H}_{2} \mathrm{O}\right)_{2}$ concentration (determined at $13^{\circ}$ and $80 \%$ relative humidity), we calculate an optical depth of approximately 0.2 , as opposed to the measured value reported by Daniel et al. of 0.02. This confirms our suspicion that the predicted dimer absorption cross sections, ${ }^{51}$ based on the rigid and relatively inaccurate RWK potential, ${ }^{19}$ must be in error, since those values combined with our predicted dimer concentrations would actually yield measurable absorptions. A correct treatment of the dimer $\mathrm{O}-\mathrm{H}$ overtone absorptions would require a full 12D treatment of the VRT dynamics, which is currently being developed by Leforestier et al. ${ }^{52}$

Additional Thermodynamic Properties. Once the canonical partition function and $K_{\mathrm{P}}(T)$ have been determined, a number of other useful thermodynamic properties of $\left(\mathrm{H}_{2} \mathrm{O}\right)_{2}$ can easily be determined. First, we can assume that $\left(\mathrm{H}_{2} \mathrm{O}\right)_{2}$ behaves like a perfect gas without mutual attraction between dimers. This is justified by the fact that in our treatment of the dimer we have assumed that it does not interact with its surroundings and we have not included any coupling with dissociative states. Thus, the following equations can be used to calculate the thermodynamic properties of the dimer: ${ }^{53}$

$$
\begin{gathered}
G_{i}=R T\left(\ln \frac{N \lambda^{3}}{V}-\ln Q_{i}\right) \\
H_{i}=R T\left(\frac{5}{2}+T \frac{\mathrm{d} \ln Q_{i}}{\mathrm{~d} T}\right) \\
S_{i}=R\left[\ln \left(\frac{V}{N \lambda^{3}} \mathrm{e}^{5 / 2}\right)+\ln Q_{i}+T \frac{\mathrm{d} \ln Q_{i}}{\mathrm{~d} T}\right] \\
C_{P}=R\left(\frac{5}{2}+\frac{\mathrm{d}}{\mathrm{d} T} T^{2} \frac{\mathrm{d} \ln Q_{i}}{\mathrm{~d} T}\right) \\
C_{V}=R\left(\frac{3}{2}+\frac{\mathrm{d}}{\mathrm{d} T} T^{2} \frac{\mathrm{d} \ln Q_{i}}{\mathrm{~d} T}\right)
\end{gathered}
$$

In the above equations, $i=1$ or 2 , corresponding to the monomer or dimer, respectively, $G$ is the Gibbs free energy, $H$ is the enthalpy, $S$ is the entropy, and $C_{P}$ and $C_{V}$ are the constant pressure and constant volume heat capacity, respectively. $Q_{i}$ is the canonical partition function from eq $1, \lambda_{i}$ is the thermal wavelength from eq 5 , and $V$ is the total molar volume. Due to the ambiguity in treating $V, \Delta G^{\circ}$ and $\Delta S^{\circ}$ for dimerization were calculated indirectly. First, $\Delta G^{\circ}$ was calculated by using the well-known equation $\Delta G^{\circ}=-R T \ln K$. Next, $\Delta H^{\circ}$ was determined by using eq 9 and the relation $\Delta H=H_{2}-2 H_{1}$. Finally, $\Delta S^{\circ}$ was calculated by the relation, $\Delta S=T(\Delta H-\Delta G)$. The results for all thermodynamic functions ${ }^{54}$ are shown in Figure 5.

Our thermodynamic results once again agree reasonably well with the sparse experimental estimates available. Our value for $\Delta G^{\circ}$ agrees quite well with the results from Curtiss et al. ${ }^{24}$ (within ca. $10 \%$ ), but our calculated value for $\Delta H^{\circ}$ at $358 \mathrm{~K}$ deviates by roughly $18 \%$ and for $\Delta S^{\circ}$ at $358 \mathrm{~K}$ deviates by ca. $15 \%$. That being said, the disagreement between our results is expected; as we have stated, $K_{\mathrm{P}}$ measured by Curtiss et al. is to be considered an upper limit for the correct experimental value, so the experimental estimate of $\Delta G^{\circ}$ is correspondingly the lower limit. $\Delta H^{\circ}$ and $\Delta S^{\circ}$ are much less precise than our results since ours were determined quite rigorously, rather than being estimated by a van't Hoff plot. To the best of our knowledge, there are no $\left(\mathrm{H}_{2} \mathrm{O}\right)_{2}$ experimental or theoretical data on the heat capacities with which to compare.

The Role of Quasi-Bound States. In a recent article, ${ }^{26}$ Schenter et al. analyze our original $K_{\mathrm{P}}(T)$ calculation ${ }^{25}$ with the VRT(ASP-W)I IPS (the first fitting of ASP-W ${ }^{18}$ ). Using a rigidrotor harmonic oscillator (RRHO) approximation for $\left(\mathrm{H}_{2} \mathrm{O}\right)_{2}$, they performed classical and quantum density of states calculations and find that there exists a substantial number of states above dissociation, calling into question the treatment of quasibound states in our calculations. As a result, they decided to test the role of quasi-bound states in the partition function by performing the calculation for the dimer with the RRHO potential model and by imposing four different constraints: (1) The available phase space is restricted to those states with total energies below the dissociation energy, $D$. This is the equivalent of what was used for our calculations with both VRT(ASPW)I ${ }^{25}$ and VRT(ASP-W)III. (2) The total energy is separated into rotational and vibrational parts; the vibrational energy is restricted to be below $D$, but the rotational part is unrestricted. This constraint is strictly equivalent to $J$-shifting. ${ }^{39-41}$ In the absence of collisions (such as in our calculations), $J$ and $K$ will be good quantum numbers, that is, the rotational energy of the system is contained entirely within the dimer. Hence, this constraint may lead to more accurate results than the first. (3) Configuration space is constrained so that the vibrational motion is restricted to lie between the harmonic turning points of the potential. The vibrational partition function is then treated via a harmonic constraint. Hence, vibrational states above dissociation can be included. (4) Configuration space is constrained so the center of mass distances between the two monomers is restricted to be less than the distance $R_{\text {cut }}$.

Schenter et al. find that depending on the constraint, in the temperature range from $200-500 \mathrm{~K}$, the values of the water dimer partition function vary by over 2 orders of magnitude. They then conclude that the choice of how dissociative states are treated is just as important as having an accurate quantum mechanical treatment in evaluating the partition function.

Although the extreme oscillations of the value of the dimer partition function in their calculations are clearly due to their use of the primitive RRHO model, their questioning of the role of quasi-bound states is still quite relevant. Hence, we will analyze our $K_{\mathrm{P}}(T)$ results in terms of the constraints mentioned above. The first constraint is perhaps the most stringent and has already been explored by our results described above. The effect of the fourth constraint, using a cutoff radius $R_{\text {cut }}$ to define the monomers, has been discussed above, wherein the $\mathrm{O}-\mathrm{O}$ separation for our calculations was varied from a range of 4-6 au to a range of 4-25 au. We found that our results varied by less than 5\%, indicating that the radial constraint had little effect on our calculations. Consequently, here we will explore the effects of the second and third constraints.

The second rotational energy constraint was tested by a simple change in our code wherein rotational energy corrections calculated via eq 3 were allowed to produce rovibrational states with energies greater than dissociation (i.e., $>0 \mathrm{~cm}^{-1}$ ), whereas previously we had discarded such states from our calculations. The same $J=0$ vibrational line list was used as before, and $K_{\mathrm{P}}$ was recalculated for each of the temperatures shown in Figure 1. The results are shown in Figure 6.

It is evident that removing the rotational energy constraint does increase the value of $K_{\mathrm{P}}(T)$ by ca. $20 \%$ and in fact makes our results much closer to the experimental ones. ${ }^{24}$ This is not entirely unexpected considering, as stated above, that in the absence of collisions, even rotational energy levels above 

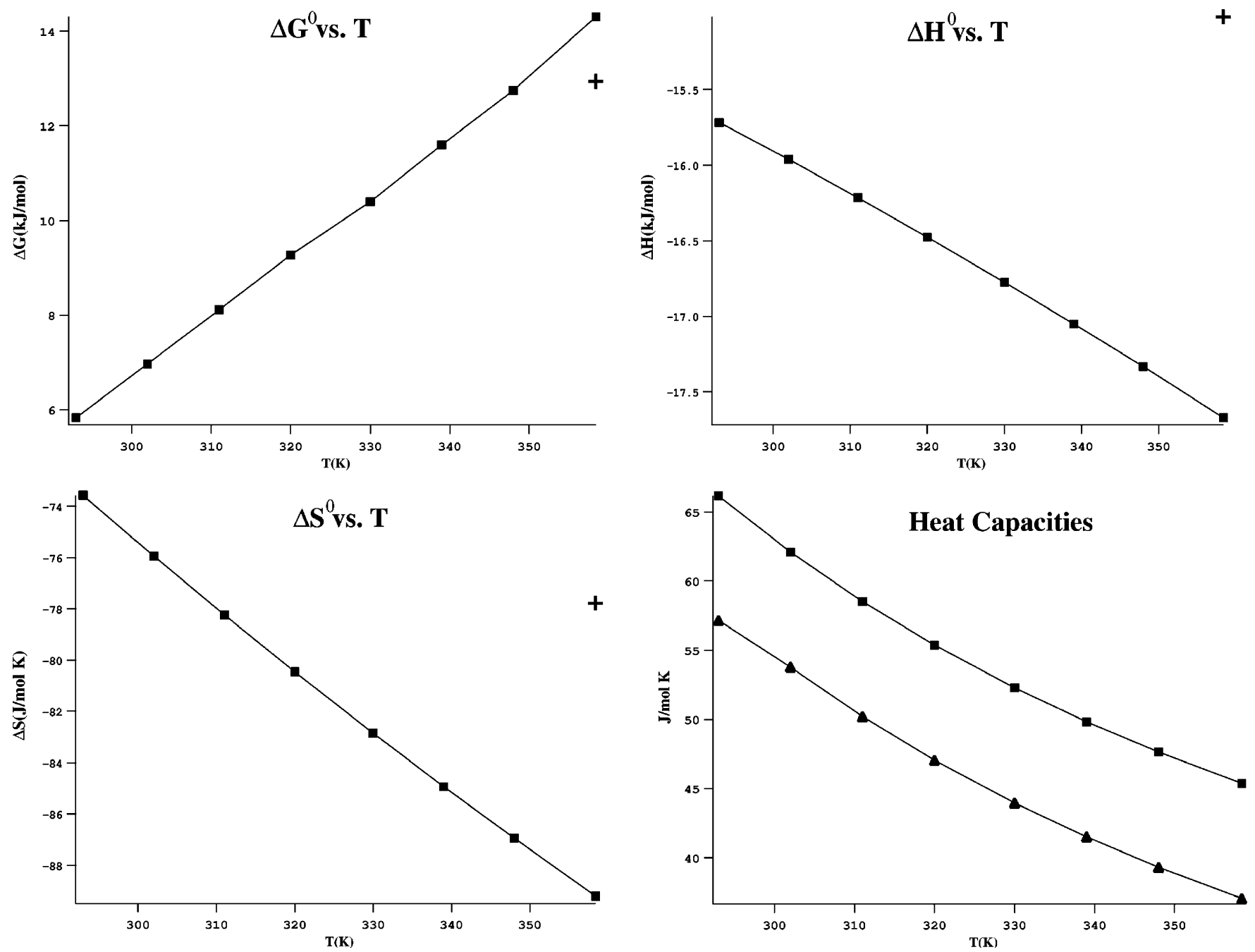

Figure 5. Thermodynamic functions for $\left(\mathrm{H}_{2} \mathrm{O}\right)_{2}$. The squares correspond to our results for $\Delta G^{\circ}, \Delta H^{\circ}$, and $\Delta S^{\circ}$, and the plus (+) on the plot corresponds to the estimates made from reference. ${ }^{24} \Delta G^{\circ}$ from the results of Curtiss et al. was calculated by us from $K_{\mathrm{P}}(T=358.4 \mathrm{~K})$ and $\Delta G^{\circ}=$ $-R T \ln K . \Delta H^{\circ}$ and $\Delta S^{\circ}$ were determined by Curtiss et al. via a van't Hoff plot over the temperature range of 358.4 to $386.4 \mathrm{~K}$. For the heat capacities, the closed squares correspond to $C_{P}$ and the closed triangles to $C_{V}$.

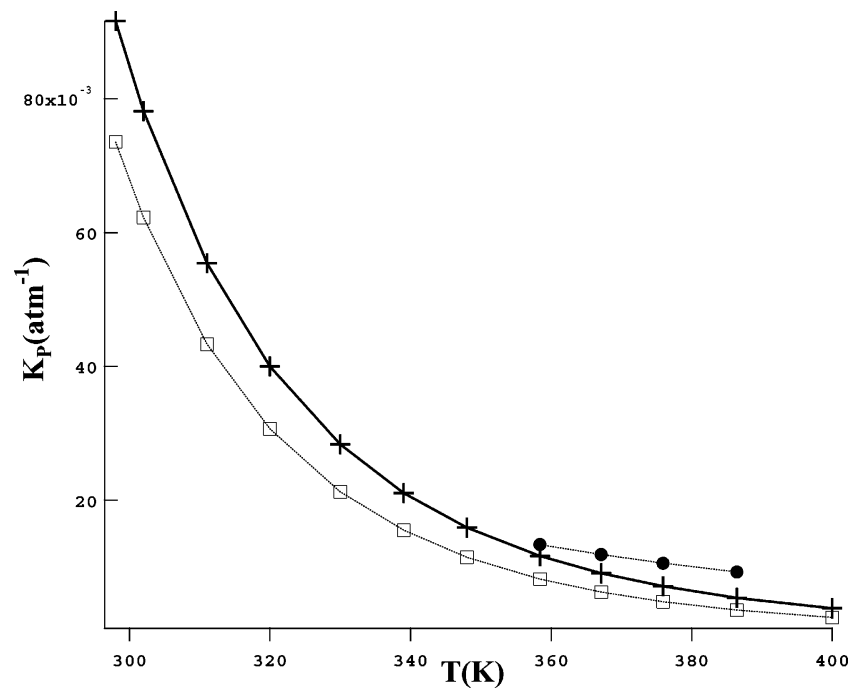

Figure 6. $K_{\mathrm{P}}(T)$ calculated from VRT(ASP-W)III with the rotational energy unconstrained in the partition function calculation. The solid line with pluses corresponds to the unconstrained results, the open squares correspond to the results presented in Figure 1, and the solid circles correspond to the experimental results. ${ }^{24}$

dissociation are contained within the dimer. However, the increase in $K_{\mathrm{P}}(T)$ is hardly as dramatic as that found by Schenter et $a{ }^{26}$ As we have stated, the RRHO model for the dimer is much too primitive, considering the complex and highly anharmonic nature of the water dimer IPS. Concerning their density of states calculation, it is quite likely that the density of states of VRT(ASP-W)III above dissociation is quite high at the temperatures investigated. However, the Boltzmann weighting of such states is relatively small and, as a result, the effect on $K_{\mathrm{P}}(T)$ is fairly small. This is highlighted in Figure 7. As is shown, at $298 \mathrm{~K}$ the value of $K_{\mathrm{P}}$ calculated with full energetic constraints (both rotational and vibrational below dissociation) is slightly more than $80 \%$ of the value of $K_{\mathrm{P}}$ determined without rotational energy constraint (but with vibrational energy constrained to below dissociation). At the point wherein the rotational energy cutoff is raised to $700 \mathrm{~cm}^{-1}$ above dissociation, $K_{\mathrm{P}}$ is within ca. $1 \%$ of the unconstrained value. Hence, our previous results ${ }^{25}$ were converged to within $80 \%$ of the results determined with inclusion of quasi-bound states.

It was decided to test the third constraint described by Schenter et al. by changing the vibrational energy cutoff in our calculations. Since it is too computationally expensive to recalculate our $J=0$ vibrational line list so that it contains states above dissociation, the convergence of $K_{\mathrm{P}}(T)$ was tested by truncating the line list so that the highest states were ca. $200 \mathrm{~cm}^{-1}$ below dissociation (rather than just below dissociation as before). In addition, the calculations were repeated without 


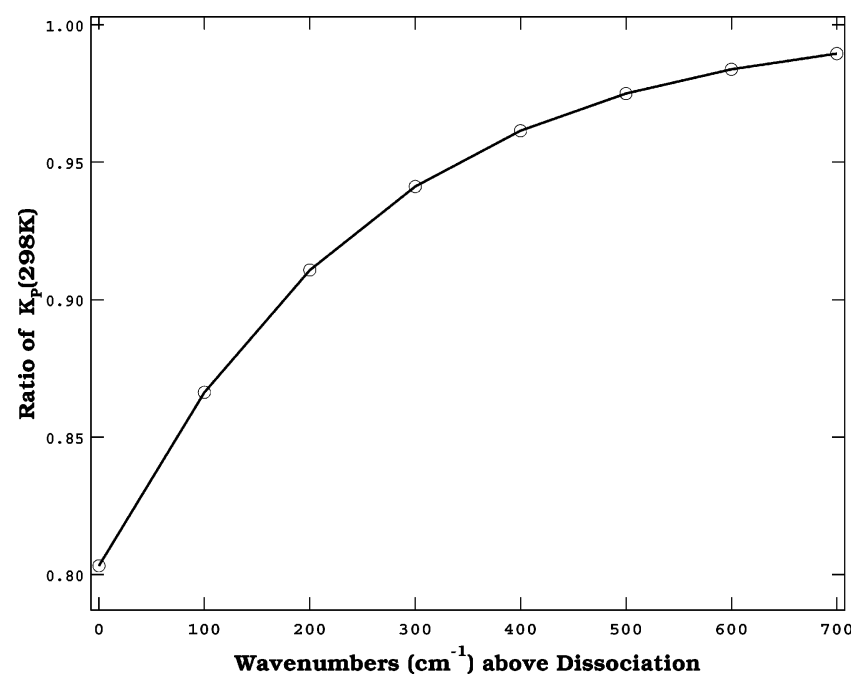

Figure 7. The ratio of $K_{\mathrm{P}}(298 \mathrm{~K})$ calculated with varying levels of rotational energy constraint to $K_{\mathrm{P}}(298 \mathrm{~K})$ calculated with the constraint removed.

TABLE 2: Comparison of Various Theoretical Values for $\left(\mathrm{H}_{2} \mathrm{O}\right)_{2}$ Partial Pressure to VRT(ASP-W)III at $100 \%$ Relative Humidity and 298 K, unless Noted Otherwise

\begin{tabular}{lc}
\hline \multicolumn{1}{c}{ source } & $\begin{array}{c}\text { atmospheric dimer } \\
\text { abundance (Torr) }\end{array}$ \\
\hline VRT(ASP-W)III (this reference) & $54 \times 10^{-3}$ \\
Slanina et al. ${ }^{47}$ & $41 \times 10^{-3}$ \\
Suck et al. $^{13}$ & $11 \times 10^{-3}$ \\
Mhin et al. $^{56}$ & $21 \times 10^{-3}$ \\
Braun and Leidecker & 54 \\
Pfeilsticker et al. $(292.4 \mathrm{~K})^{22}$ & $18 \times 10^{-3}$ \\
\end{tabular}

constraining the rotational energies to be below $0 \mathrm{~cm}^{-1}$, as discussed before. It was found that at $298 \mathrm{~K} K_{\mathrm{P}}$ evaluated with the truncated vibrational line list deviated from the calculation with the untruncated list by less than $5 \%$. Hence, it is clear that with the inclusion of vibrational states up to dissociation, the vibrational part of the partition function is converged. Consequently, inclusion of vibrational states above dissociation will have no appreciable effects on our calculations.

\section{Discussion}

The dimer concentrations predicted here for high humidity conditions may be sufficient to influence several important atmospheric chemical reactions and perhaps for absorption of sunlight. Comparison of several previous theoretical results for $P_{\left(\mathrm{H}_{2} \mathrm{O}\right)_{2}}$ and the experimental results from Pfeilsticker et al. ${ }^{22}$ is made to our new results from VRT(ASP-W)III in Table 2. All of the previous theoretical results shown were calculated within a simplistic harmonic oscillator approximation, and the largest $P_{\left(\mathrm{H}_{2} \mathrm{O}_{2}\right.}$ obtained is equal to the predicted value from VRT(ASPW)III, which is ca. 3 times greater than the recent experimental measurement of Pfeilsticker et al. ${ }^{22}$ The discrepancies in the previous theoretical calculations highlight the necessity for a rigorous anharmonic treatment of the dimer dynamics.

We have also calculated additional thermodynamic properties for $\mathrm{H}_{2} \mathrm{O}$ dimerization, which represents the first time that such properties, like the heat capacities, have been calculated at this level of accuracy. While the experimental data for such functions are sparse, the results presented here provide benchmarks for future theoretical and experimental studies. In addition, we have been able to thoroughly address concerns regarding the exclusion of quasi-bound states in our previous calculations. Although the effects of these quasi-bound states has been greatly overestimated by Schenter et al., ${ }^{26}$ they do induce changes of ca. $20 \%$ for the temperature range of interest to atmospheric scientists.

The high concentrations of atmospheric water dimers predicted by our calculations have been confirmed by the very recent measurements of $\left(\mathrm{H}_{2} \mathrm{O}\right)_{2}$ overtone vibrational transitions in the atmosphere by Pfeilsticker et al. ${ }^{22}$ Interestingly, these measurements yield $K_{\mathrm{P}}(T)$ values that are below both those that we originally reported ${ }^{25}$ and those reported herein. The authors also extrapolate the $K_{\mathrm{P}}$ measurements of Curtiss et al. from 375 $\mathrm{K}^{24}$ to the temperature at which their measurements were conducted $(292.4 \mathrm{~K})$ by assuming that the values of $\Delta H^{\circ}$ and $\Delta S^{\circ}$ reported by Curtiss et al. are constant over temperature and then using the relation $K_{\mathrm{P}}(T)=\exp \left[\left(-\Delta H^{\circ}+T \Delta S^{\circ}\right) /(R T)\right]$. Their results show that their measured values of $K_{\mathrm{P}}(T)$ are within $10 \%$ of the above extrapolation and lie roughly between our original results from VRT(ASP-W) ${ }^{25}$ and those of Vaida and Headrick, ${ }^{16}$ which were determined via the aforementioned simple harmonic approximation.

The results of Pfeilsticker et al. are very exciting in that they are the first reported measurements of $\left(\mathrm{H}_{2} \mathrm{O}\right)_{2}$ atmospheric absorption. However, these measurements should be viewed with cautious optimism and must be substantiated by further experiments. The assignment of the observed absorption measurements to the water dimer is based largely on the theoretical results of Low and Kjaergaard, ${ }^{23}$ in which the fundamental and overtone $\mathrm{OH}$-stretching vibrational spectra were calculated via a harmonically coupled anharmonic oscillator (HCAO) local mode model and ab initio dipole moment functions. The HCAO method thus describes the water dimer by modeling the intermolecular motion as two Morse oscillators that are harmonically coupled. Pfeilsticker et al. conclude that they have measured atmospheric water dimer absorption since the "band center" of their measurement is within the wavelength error range for the HCAO calculated $|0\rangle_{a}|4\rangle_{b}$ water dimer $\mathrm{OH}$ stretching transition. Considering, in particular, the very strong degree of anharmonicity of the water dimer IPS and the high density of vibration-tunneling states, harmonic coupling is generally speaking a poor model of intermolecular modes. Thus, the HCAO model for the water dimer is still somewhat crude, and the results derived therein must be regarded with caution. Nevertheless, the fundamental $\mathrm{OH}$-stretching vibrations predicted for $\left(\mathrm{H}_{2} \mathrm{O}\right)_{2}$ via $\mathrm{HCAO}$ correspond well with existing experimental results. ${ }^{23}$ Also, the measured absorptions from Pfeilsticker et al. are proportional to the square of the partial pressure of water monomer, which is what is expected for the water dimer (see eq 4). Experimental measurements would clearly benefit from improved calculation of the $\left(\mathrm{H}_{2} \mathrm{O}\right)_{2}$ overtone absorption spectrum, and efforts in this direction are currently underway. ${ }^{45}$

In addition, the assumption of isothermicity of $\Delta H$ and $\Delta S$ by Pfeilsticker et al. is not particularly well-supported. Indeed, our calculations of these properties indicate that they vary significantly over temperature. For example, at $375 \mathrm{~K}$, our results from VRT(ASP-W)III, with corrections for inclusion of quasi-bound states, show that $\Delta H^{\circ}=-15.66 \mathrm{~kJ} / \mathrm{mol}$ and $\Delta S^{\circ}$ $=-82.65 \mathrm{~J} / \mathrm{mol} \mathrm{K}$, both of which are well within ca. $6 \%$ of the values determined by Curtiss et al. and well within the error bounds published therein. ${ }^{24}$ Using the same expression as that used by Pfeilsticker et al. and the above results for $\Delta H^{\circ}$ and $\Delta S^{\circ}$, we can estimate $K_{\mathrm{P}}(292.4 \mathrm{~K})$ to be $0.0302 \mathrm{~atm}^{-1}$. However, our more rigorous partition function calculation at $292.4 \mathrm{~K}$ yields results of $\Delta H^{\circ}=-14.24 \mathrm{~kJ} / \mathrm{mol}, \Delta S^{\circ}=-66.68 \mathrm{~J} / \mathrm{mol} \mathrm{K}$, and $K_{\mathrm{P}}(292.4 \mathrm{~K})=0.1151 \mathrm{~atm}^{-1}$. Thus, slight variations in $\Delta H^{\circ}$ and 
$\Delta S^{\circ}$ (ca. $10-15 \%$ in this example) can have large ramifications in the approximation used by Pfeilsticker et al. due to the exponential dependence of $K_{\mathrm{P}}(T)$. Consequently, their comparison to the results of Curtiss et al. is not quite as compelling, and the true value of $K_{\mathrm{P}}(T)$ at $292.4 \mathrm{~K}$ most likely is higher than they predict. However, it should be noted that due to the sparsity of the data from Curtiss et al., the assumption of isothermicity of $\Delta H^{\circ}$ and $\Delta S^{\circ}$ was the only way in which Pfeilsticker et al. could make the above comparisons.

Future work could involve improvement of our thermodynamics calculations via the use of path integral Monte Carlo (PIMC). This technique has already been successfully applied to $\left(\mathrm{H}_{2} \mathrm{O}\right)_{2}{ }^{55}$ but with the use of models that do not have the spectroscopic accuracy of ours. PIMC has the advantage of not requiring any assumption about rotational constants nor a priori knowledge about the water dimer symmetry, thus transcending these limitations of our current approach. Hence, such calculations could, in principle, directly determine the most accurate value of the canonical partition function that the VRT(ASPW)III IPS is capable of producing.

Acknowledgment. This work was supported by the Experimental Physical Chemistry Program of the NSF and by the International Grants Program of the NSF and CNRS.

\section{References and Notes}

(1) Daniel, J. S.; Solomon, S.; Sanders, R. W.; Portmann, R. W.; Miller, D. C.; Madsen, W. J. Geophys. Res., [Atmos.] 1999, 104, 16785-16791. 2018.

(2) Chylek, P.; Geldart, D. J. W. Geophys. Res. Lett. 1997, 24, 2015-

(3) Goss, L. M.; Vaida, V. Stratospheric Processes and Their Role in Climate: (SPARC): Proceedings of the First SPARC General Assembly; World Meteorological Organization: Melbourne, Australia, 1996.

(4) Devir, A. D.; Neumann, M.; Lipson, S. G.; Oppenheim, U. P. Opt. Eng. 1994, 33, 746-750.

(5) Akhmatskaya, E. V.; Apps, C. J.; Hillier, I. H.; Masters, A. J.; Watt, N. E.; Whitehead, J. C. Chem. Commun. 1997, 7, 707.

(6) Loerting, T.; Liedl, K. R. Proc. Natl. Acad. Sci. U.S.A. 2000, 97, 8874-8878.

(7) Lovejoy, E. R.; Hanson, D. R.; Huey, L. G. J. Phys. Chem. 1996, 100, 19911-19916.

(8) Jayne, J. T.; Poschl, U.; Chen, Y. M.; Dai, D.; Molina, L. T.; Worsnop, D. R.; Kolb, C. E.; Molina, M. J. J. Phys. Chem. A 1997, 101, 10000-10011.

(9) Slanina, Z.; Crifo, J. F. Int. J. Thermophys. 1992, 13, 465-476. 1902.

(10) Aloisio, S.; Francisco, J. S. J. Phys. Chem. A 1998, 102, 1899-

(11) Aloisio, S.; Francisco, J. S.; Friedl, R. R. J. Phys. Chem. A 2000, 104, 6597-6601.

(12) Vaida, V.; Daniel, J. S.; Kjaergaard, H. G.; Goss, L. M.; Tuck, A. F. Q. J. R. Meteorol. Soc. 2001, 127, 1627-1643.

(13) Suck, S. H.; Wetmore A. E.; Chen, T. S.; Kassner, J. L. Appl. Opt. 1982, 21, 1610-1614.

(14) Pilewskie, P.; Valero, F. P. J. Science 1995, 267, 1626-1629.

(15) Munoz-Caro, C.; Nino, A. J. Phys. Chem. A 1997, 101, 41284135 .

(16) Vaida, V.; Headrick, H. E. J. Phys. Chem. A 2000, 104, 54015412.

(17) Evans, G. T.; Vaida, V. J. Chem. Phys. 2000, 113 (16), 66256659.
(18) Fellers, R. S.; Leforestier, C.; Braly, L.; Brown, M. G.; Saykally, R. J. Science 1999, 284, 945 .

(19) Fellers, R. S.; Braly, L.; Saykally, R. J.; Leforestier, C. J. Chem. Phys. 1999, 110, 6306.

(20) Goldman, N.; Fellers, R. S.; Brown, M. G.; Braly, L. B.; Keoshian, C. J.; Leforestier, C.; Saykally, R. J. J. Chem. Phys. 2002, 116, 1014810163.

(21) Hill, C.; Jones, R. L. J. Geophys. Res., [Atmos.] 2000, 105, $9421-$ 9428 .

(22) Pfeilsticker, K.; Lotter, A.; Peters, C.; Bösch, H. Science 2003, $300,2078$.

(23) Low, G. R.; Kjaergaard, H. G. J. Chem. Phys. 1999, 110 (18), 9104.

(24) Curtiss, L. A.; Frurip, D. J.; Blander, M. J. Chem. Phys. 1979, 71 (6), 2703-2711.

(25) Goldman, N.; Fellers, R. S.; Leforestier, C.; Saykally, R. J. J. Phys. Chem. A 2001, 105, 515-519.

(26) Schenter, G. K.; Kathmann, S. M.; Garrett, B. C. J. Phys. Chem. A 2002, 106, 1557-1566.

(27) Smith, B. J.; Swanton, D. J.; Pople, J. A.; Schaefer, H. F.; Radom, L. J. Chem. Phys. 1990, 92 (2), 1240-1247.

(28) Scheiner, S. Annu. Rev. Phys. Chem. 1994, 45, 23-56.

(29) Millot, C.; Stone, A. J. Mol. Phys. 1992, 77 (3), 439-462.

(30) Saykally, R. J.; Blake, G. A. Science 1993, 259, 1570-1575.

(31) Keutsch, F. N.; Saykally, R. J. Proc. Natl. Acad. Sci. U.S.A. 2001, 98, 10533

(32) Braly, L. B.; Cruzan, J. D.; Liu, K.; Fellers, R. S.; Saykally, R. J. J. Chem. Phys. 2000, 112, 10293.

(33) Braly, L. B.; Liu, K.; Brown, M. G.; Keutsch, F. N.; Fellers, R. S.; Saykally, R. J. J. Chem. Phys. 2000, 112, 10314.

(34) Busarow, K. L.; Cohen, R. C.; Blake, G. A.; Laughlin, K. B.; Lee, Y. T.; Saykally, R. J. J. Chem. Phys. 1989, 90, 3937-3943.

(35) Groenenboom, G. C.; Mas, E.; Bukowski, R.; Szalewicz, K.; Wormer, P. E. S.; van der Avoird, A. Phys. Rev. Lett. 2000, 84, 4072.

(36) Leforestier, C.; Braly, L. B.; Liu, K.; Elrod, M. J.; Saykally, R. J. J. Chem. Phys. 1997, 106 (20), 8527-8544.

(37) Harris, D. O.; Engerholm, G. G.; Gwinn, W. D. J. Chem. Phys. 1965, 43 (5), 1515-1517.

(38) Dyke, T. R. J. Chem. Phys. 1977, 66 (2), 492-497.

(39) Sun, Q.; Bowman, J. M.; Schatz, G. C.; Sharp, J. R.; Connor, J. N. L. J. Chem. Phys. 1990, 92 (3), 1677.

(40) Bowman, J. M. J. Phys. Chem. 1991, 95 (13), 4960-4968.

(41) Bowman, J. M.; Shnider, H. M. J. Chem. Phys. 1999, 110 (9), 4428.

(42) Harris, G. J.; Viti, S.; Mussa, H. Y.; Tennyson, J. J. Chem. Phys.

1998, 109, 7197-7204.

(43) Viti, S. Ph.D. Thesis, University College, London, 1997.

(44) Polyansky, O. L.; Jennson, P.; Tennyson, J. J. Chem. Phys. 1996, 105, 6490-6497.

(45) Leforestier, C. Private communication.

(46) Braly, L. B. The Intermolecular Vibrations of the Water Dimer.

Ph.D. Thesis, University of California, Berkeley, CA, 1999.

(47) Slanina, Z.; Uhlik, F. Int. J. Thermophys. 1992, 13, 303-313.

(48) Xantheas, S. S. J. Chem. Phys. 1996, 104 (21), 8821-8824.

(49) Feyereisen, M. W.; Feller, D.; Dixon, D. A. J. Phys. Chem. 1996, 100, 2993-2997.

(50) Mas, E. M.; Szalewicz, K. J. Chem. Phys. 1996, 104 (19), 76067614.

(51) Tso, H. C. W.; Geldart, D. J. W.; Chylek, P. J. Chem. Phys. 1998, 108 (13), 5319-5329.

(52) Leforestier, C.; Gatti, F.; Fellers, R. S.; Saykally, R. J. J. Chem. Phys. 2002, 117, 8710-8722.

(53) Mayer, J. E.; Mayer, M. G. Staistical Mechanics, 2nd ed.; WileyInterscience: New York, 1977.

(54) Therochemical results in tabular form are available from the authors upon request.

(55) Schenter, G. K. J. Chem. Phys. 1998, 108, 6222-6232.

(56) Mhin, B. J.; Lee, S. J.; Kim, K. S. Phys. Rev. A 1993, 93 (5), 37643770 .

(57) Braun, C.; Leidecker, H. J. Chem. Phys. 1974, 61 (8), 3104-3113. 